University of New Orleans

ScholarWorks@UNO

$12-15-1985$

\title{
Total refraction at oblique incidence by a transparent bilayer coating on a high-index transparent or absorbing substrate
}

\author{
R. M.A. Azzam \\ University of New Orleans, razzam@uno.edu \\ Karim Javily
}

Follow this and additional works at: https://scholarworks.uno.edu/ee_facpubs

Part of the Electrical and Electronics Commons, and the Optics Commons

\section{Recommended Citation}

R. M. A. Azzam and Karim Javily, "Total refraction at oblique incidence by a transparent bilayer coating on a high-index transparent or absorbing substrate," Appl. Opt. 24, 4454-4459 (1985)

This Article is brought to you for free and open access by the Department of Electrical Engineering at ScholarWorks@UNO. It has been accepted for inclusion in Electrical Engineering Faculty Publications by an authorized administrator of ScholarWorks@UNO. For more information, please contact scholarworks@uno.edu. 


\title{
Total refraction at oblique incidence by a transparent bilayer coating on a high-index transparent or absorbing substrate
}

\author{
R. M. A. Azzam and Karim Javily
}

\begin{abstract}
Transparent bilayer coatings that produce total refraction (TR) of obliquely incident monochromatic light into an underlying substrate are considered. When the substrate is transparent, it is shown that TR takes place without any accompanying change of polarization. Totally refracting bilayers are realizable in the IR where high-refractive-index substrates are available. This is illustrated by a $\mathrm{BaF}_{2}-\mathrm{ZnSe}$ bilayer on a $\mathrm{Ge}$ substrate at a 10.6- $\mu \mathrm{m}\left(\mathrm{CO}_{2}\right.$-laser) wavelength and $45^{\circ}$ angle of incidence. Limited changes of the angle of incidence, wavelength, and refractive indices and thicknesses of the two films of the bilayer are introduced, and their effects on the condition of TR are determined. TR (hence absorption) is also possible for absorbing (semiconductor or metallic) substrates using transparent bilayers of films of nonquarter-wave optical thickness, as is further demonstrated in this paper.
\end{abstract}

\section{Introduction}

For a given transparent or absorbing substrate at a given angle of (oblique) incidence, it is possible to suppress completely the reflection of parallel $p$-or perpendicularly $s$-polarized incident monochromatic light using a transparent single-layer coating. ${ }^{1}$ However, when the incident light has nonzero $p$ and $s$ components (e.g., with equal mix as in the case of unpolarized or circularly polarized light), the reflectance of the surface can only be reduced by a singlelayer coating to a nonzero minimum. ${ }^{2,3}$ Complete and simultaneous antireflection of the $p$ and $s$ polarizations at oblique incidence (a condition that we shall conveniently refer to as total refraction) requires at least two layers, as was first noted by Baumeister. ${ }^{4}$ When the substrate is transparent, each one of the two layers is of quarter-wave optical thickness at the given angle of incidence. For a glass substrate (with refractive index of 1.5 ), the required refractive index of the outer layer (in contact with the air) is too low (1.08) to make total refraction using a bilayer practical. ${ }^{4}$

In this paper we examine realizable bilayer coatings on a high-index (e.g., Ge) substrate that produce total

The authors are with University of New Orleans, Lakefront, Department of Electrical Engineering, New Orleans, Louisiana 70148.

Received 12 July 1985.

0003-6935/85/244454-06\$02.00/0.

(C) 1985 Optical Society of America. refraction (TR) of incident (IR) monochromatic light at oblique incidence. The sensitivity of the design to changes of the angle of incidence, wavelength, film refractive indices, and film thicknesses is considered. We also prove that TR occurs without any change of polarization. Furthermore, we find that TR (hence total absorption) continues to be possible with transparent bilayer coatings when the substrate is absorbing (e.g., semiconducting or metallic) over a wide range of the substrate extinction coefficient. In the latter case, the two films of the bilayer no longer have quarterwave optical thicknesses.

\section{Bilayer Coatings that Produce Total Refraction}

Consider a monochromatic light beam of wavelength $\lambda$ traveling in an ambient (medium 0 , usually air) which is reflected at an angle $\phi$ by two transparent thin films (films 1 and 2) on a substrate (medium 3). All (bulk and thin-flim) media are assumed to be homogeneous, optically isotropic, linear, nonmagnetic, and separated by sharp parallel-plane interfaces. The complex amplitude-reflection coefficients of such a system are given by

$$
R_{\nu}=\frac{r_{01 \nu}+r_{12 \nu} X_{1}+r_{01 \nu} r_{12 \nu} r_{23 \nu} X_{2}+r_{23 \nu} X_{1} X_{2}}{1+r_{01 \nu} r_{12 \nu} X_{1}+r_{12 \nu} r_{23 \nu} X_{2}+r_{01 \nu} r_{23 \nu} X_{1} X_{2}}, \nu=p, s,
$$

where

$$
X_{i}=\exp \left(-j 2 \pi \zeta_{i}\right), \quad i=1,2,
$$

and $r_{l m \nu}$ is Fresnel's reflection coefficient of the $l m$ interface for the $\nu$ polarization. In Eq. (2), $\zeta_{i}$ is the normalized thickness of the $i$ th film, 


$$
\zeta_{i}=d_{i} / D_{\phi i}, \quad i=1,2,
$$

where $d_{i}$ is the actual (metric) thickness, and $D_{\phi i}$ is the associated film thickness period,

$$
D_{\phi i}=\frac{\lambda}{2}\left(N_{i}^{2}-N_{0}^{2} \sin ^{2} \phi\right)^{-1 / 2}, \quad i=1,2 .
$$

$N_{i}$ is the refractive index of the $i$ th medium ( $i=$ $0,1,2,3)$. The ambient and two films are transparent, so that $N_{0}, N_{1}$, and $N_{2}$ are real, whereas the substrate is in general absorbing with complex refractive index $N_{3}$ $=n_{3}-j k_{3}$.

For a transparent substrate coated by two layers of quarterwave optical thicknesses $\left(\zeta_{1}=\zeta_{2}=1 / 2\right)$, antireflection of the $\nu$ polarization occurs when

$$
r_{01 \nu}-r_{12 \nu}+r_{23 \nu}=r_{01 \nu} r_{12 \nu} r_{23 \nu},
$$

which is readily obtained by setting the numerator of Eq. (1) equal to zero and $X_{1}=X_{2}=-1$.

For the $\nu=s$ polarization, we substitute $r_{l m s}=\left(S_{l}-\right.$ $\left.S_{m}\right) /\left(S_{l}+S_{m}\right)$ in Eq. (5) and obtain (after considerable simplification)

where

$$
S_{0} S_{2}^{2}=S_{1}^{2} S_{3}
$$

$$
S_{i}=\left(N_{i}^{2}-N_{0}^{2} \sin ^{2} \phi\right)^{1 / 2}, \quad i=0,1,2,3 .
$$

Similarly, for the $\nu=p$ polarization, substitution of $r_{l m p}=\left(N_{m}^{2} S_{l}-N_{l}^{2} S_{m}\right) /\left(N_{m}^{2} S_{l}+N_{l}{ }^{2} S_{m}\right)$ into Eq. (5) yields

$$
N_{1}^{4} N_{3}^{2} S_{0} S_{2}^{2}=N_{0}^{2} N_{2}{ }^{4} S_{1}{ }^{2} S_{3}
$$

It is convenient to define the relative refractive indices, $n_{i}=N_{i} / N_{0}$ (here $i=1,2,3$ ), and introduce the variable $u=\sin ^{2} \phi$.

Concomitant antireflection of the $p$ and $s$ polarizations (hence total refraction) is possible when Eqs. (6) and (8) are satisfied simultaneously. When Eq. (6) is satisfied, Eq. (8) will also be satisfied if $N_{1}{ }^{4} N_{3}{ }^{2}=$ $\mathrm{N}_{0}{ }^{2} \mathrm{~N}_{2}^{4}$, or

$$
n_{2}{ }^{4}=n_{1}{ }^{4} n_{3}{ }^{2} .
$$

Equation (6) [with $S_{i}$ given by Eq. (7)] and Eq. (9) are sufficient to determine the required refractive indices $n_{1}$ and $n_{2}$ of the two films for given $n_{3}$ and $\phi$. By manipulating these equations, we get the following solution:

$$
n_{1}^{2}=\left\{\left(n_{3}+u\right)+\left[\left(n_{3}+u\right)^{2}-\left(n_{3}+1\right)^{2} u\right]^{1 / 2}\right\} /\left(n_{3}+1\right) .
$$

After determining $n_{1}$ from Eq. (10), $n_{2}$ is subsequently obtained from Eq. (9).

It is instructive to consider some limit of Eq. (10). When $n_{3} \gg 1$ (very-high-index substrate), we obtain

$$
n_{1}=\sqrt{2} \cos (\phi / 2),
$$

which is independent of $n_{3}$. Thus the refractive index of the outer layer (next to air) is always $<\sqrt{2=1.414}$. As $\phi \rightarrow 0, u \rightarrow 0$, and the normal-incidence limit of $n_{1}{ }^{2}$ from Eq. (10) is

$$
n_{1}^{2}=2 n_{3} /\left(n_{3}+1\right) \text {. }
$$

As $\phi \rightarrow 90^{\circ}, u \rightarrow 1$, and the grazing-incidence limit of $n_{1}^{2}$ is found to be 1 .
The complex amplitude-transmission coefficients of the bilayer of quarterwave films $\left(X_{1}=X_{2}=-1\right)$ for the $s$ and $p$ polarizations are given by

$$
\begin{aligned}
& t_{s}=-2 S_{0} S_{1} S_{2} /\left(S_{0} S_{2}{ }^{2}+S_{1}{ }^{2} S_{3}\right), \\
& t_{p}=-2 n_{1}{ }^{2} n_{2}{ }^{2} n_{3} S_{0} S_{1} S_{2} /\left(n_{1}{ }^{4} n_{3}{ }^{2} S_{0} S_{2}{ }^{2}+n_{2}{ }^{4} S_{1}{ }^{2} S_{3}\right) .
\end{aligned}
$$

From Eq. (9) we have $n_{2}^{4}=n_{1}^{4} n_{3}^{2}=n_{1}^{2} n_{2}{ }^{2} n_{3}$. Substitution of these relations into Eq. (14) and comparing the result with Eq. (13) lead to

$$
t_{p}=t_{s}
$$

From Eq. (15) we reach the important conclusion that the state of polarization of the totally refracted light is identical to that of the incident light for all incident polarizations. In other words, the bilayer produces total refraction without change of polarization.

\section{Transparent Bilayer on a Ge Substrate Producing Total Refraction of $10.6-\mu \mathrm{m}$ Radiation at $45^{\circ}$ Angle of Incidence}

Bilayer coatings that produce total refraction at oblique incidence are practical in the IR because highindex transparent substrates are available in this part of the spectrum. As an example, consider the reflection in air $\left(N_{0}=1\right)$ of $10.6-\mu \mathrm{m} \mathrm{CO} \mathrm{CO}_{2}$-laser radiation by a Ge substrate with refractive index ${ }^{7} 4$ at $45^{\circ}$ angle of incidence. To obtain the refractive indices of the two films of the TR bilayer, $n_{3}=4$ and $u=1 / 2$ are substituted in Eq. (10) to give

$$
n_{1}=(0.9+\sqrt{0.31})^{1 / 2}=1.20697,
$$

which is identical to the index obtained from Eq. (22) of Ref. 4. A thin-film coating material with this low refractive index at $\lambda=10.6 \mu \mathrm{m}$ is $\mathrm{BaF}_{2}$, as has been reported by Kemeny. ${ }^{8}$ Equation (9) subsequently gives

$$
n_{2}=2.41394 \text {. }
$$

A possible coating material with this index ${ }^{9}$ at $10.6 \mu \mathrm{m}$ is $\mathrm{ZnSe}$. It should be remembered that the refractive indices of thin films significantly depend on conditions of their preparation which must then be controlled to attain the required values. The (least) required layer thicknesses are equal to one-half of the corresponding thickness periods; from Eqs. (4) and (16), we obtain

$$
d_{1}=2.70920 \mu \mathrm{m}, \quad d_{2}=1.14815 \mu \mathrm{m} \text {. }
$$

We now examine the behavior of this design as a function of angle of incidence $\phi$ and wavelength $\lambda$. Figure 1 shows the $p$ and $s$ intensity reflectances plotted vs $\phi$ in the range $0 \leq \phi \leq 50^{\circ}$ for the Ge substrate which is coated with the totally refracting bilayer whose properties are specified by Eqs. (16) and (17). The reflectance $\mathcal{R}_{\nu}$ for the $\nu$ polarization is determined by

$$
\mathscr{R}_{\nu}=\left|R_{\nu}\right|^{2}
$$

where $R_{\nu}$ is given by Eq. (1). For $\phi \neq 45^{\circ}$, both layers are no longer of quarterwave optical thickness, and $X_{1}$, $X_{2} \neq-1$. For $\phi<45^{\circ}, \mathcal{R}_{p}$ and $\mathscr{R}_{s}$ are $<1.4 \times 10^{-3}$, which is the normal-incidence reflectance. Increasing $\phi$ from 45 to $50^{\circ}$ increases $\mathcal{R}_{p}$ from 0 to $8.5 \times 10^{-5}$ and 


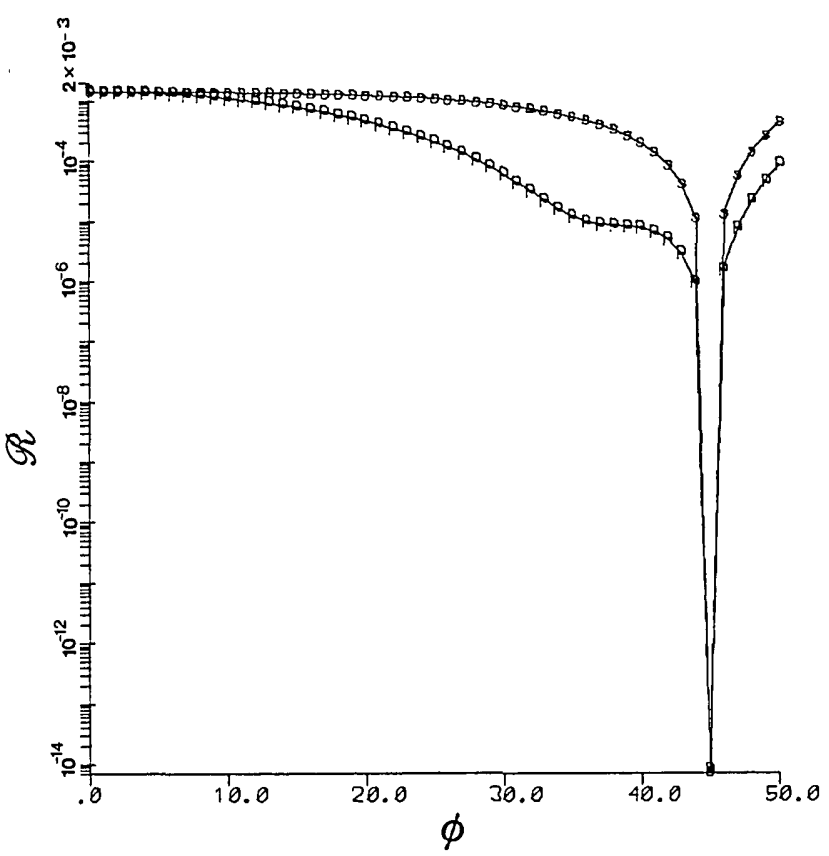

Fig. 1. $\quad p$ and $s$ intensity reflectances (on a logarithmic scale) plotted vs the angle of incidence $\phi$ (in degrees) for a bilayer of two films of refractive indices $n_{1}=1.20697$ and $n_{2}=2.41394$ and thicknesses $d_{1}=$ $2.70920 \mu \mathrm{m}$ and $d_{2}=1.14815 \mu \mathrm{m}$, respectively, on a Ge substrate of refractive index $n_{3}=4$. A $10.6-\mu \mathrm{m}\left(\mathrm{CO}_{2}\right.$-laser $)$ beam is assumed to be incident from air $\left(N_{0}=1\right)$. At $\phi=45^{\circ}, \mathcal{R}_{p}=\mathcal{R}_{s}=0$, and total refraction takes place.

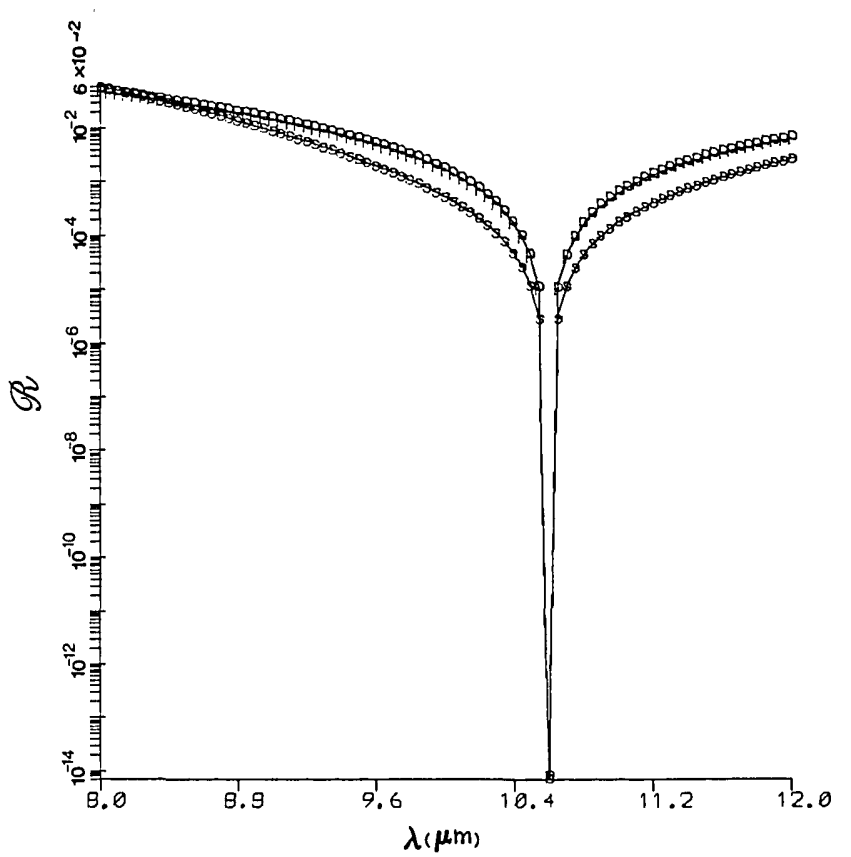

Fig. 2. $\quad p$ and $s$ intensity reflectances (on a logarithmic scale) vs wavelength $\lambda$ (in microns) at $45^{\circ}$ angle of incidence for a bilayer of two films of refractive indices $n_{1}=1.20697$ and $n_{2}=2.41394$ and thicknesses $d_{1}=2.70920 \mu \mathrm{m}$ and $d_{2}=1.14815 \mu \mathrm{m}$, respectively, on a Ge substrate of refractive index $n_{3}=4$. At the design wavelength $\lambda$ $=10.6 \mu \mathrm{m}, \mathcal{R}_{p}=\mathcal{R}_{s}=0$, and total refraction takes place from air into Ge. The effect of material dispersion is ignored.
$\mathscr{R}_{s}$ from 0 to $4.4 \times 10^{-4}$. This indicates that the condition of total refraction, which is satisfied exactly at $\phi=45^{\circ}$, continues to hold with little error over a wide range of incidence angle, $0 \leq \phi \leq 50^{\circ}$.

Figure 2 shows the variation of $\mathcal{R}_{p}$ and $\mathcal{R}_{s}$ with wavelength $\lambda$ from 8 to $12 \mu \mathrm{m}$. In this calculation, the refractive indices of the two films and of the substrate are kept constant independent of $\lambda$ (i.e., material dispersion is neglected). $\mathscr{R}_{p}=\mathscr{R}_{s}=0$ at the design wavelength of $10.6 \mu \mathrm{m}$, and both reflectances rise steeply as $\lambda$ shifts from this value. However, $\mathcal{R}_{p}$ and $\mathcal{R}_{s}$ remain below $1 \%$, for $9 \leq \lambda \leq 12 \mu \mathrm{m}$, indicating acceptable performance over an appreciable spectral band.

The sensitivity of the design to small changes $( \pm 0.1)$ of the refractive index of each of films 1 and 2 is illustrated in Figs. 3 and 4, respectively. In Fig. $3 n_{1}$ is allowed to vary by \pm 0.1 around the design index of 1.20697, although positive errors are more likely to take place in this case. In the presence of an error $\Delta n_{1}$ $= \pm 0.02$, the reflectances $\mathcal{R}_{p}$ and $\mathscr{R}_{s}$ remain $<10^{-3}$.

Figure 4 indicates that the design is more tolerant to equal errors $( \pm 0.1)$ of the higher refractive index $n_{2}$ of the inner film (that is first deposited on the Ge substrate). If $n_{2}$ is shifted by $\Delta n_{2}= \pm 0.02$, we still have $\mathcal{R}_{p}, \mathcal{R}_{s}<1.5 \times 10^{-4}$.

Finally, Figs. 5 and 6 describe the sensitivity of the design to small errors $( \pm 0.01 \mu \mathrm{m}= \pm 10 \mathrm{~nm})$ of the thickness of films 1 and 2 , respectively. Figure 5 indicates good tolerance to \pm 10 -nm errors of thickness of the outer layer, because $\mathcal{R}_{p}$ and $\mathcal{R}_{s}$ remain $<10^{-5}$. For equal errors of thickness of the inner high-index layer (film 2) Fig. 6 shows that $\mathscr{R}_{p}, \mathscr{R}_{s}<1.5 \times 10^{-3}$.

To summarize this section, we have demonstrated that totally refracting (polarization-preserving) transparent bilayers are possible on high-index substrates (e.g., Ge) in the IR (e.g., at $\lambda=10.6 \mu \mathrm{m}$ ). Furthermore, the design is found to be reasonably tolerant to changes of incidence angle, wavelength, film refractive indices, and film thicknesses.

\section{Totally Refracting Bilayers on Absorbing Substrates}

Intuitively, total refraction and absorption of obliquely incident monochromatic light should also be possible using a transparent bilayer on an absorbing substrate. However, in this case it is expected that the layers would no longer be of quarterwave optical thickness. For an absorbing substrate with complex refractive index $N_{3}=n_{3}-j k_{3}$, the analysis of Sec. II that begins with Eq. (5) is not applicable. We have adopted a numerical approach as follows. The reflectance of the bilayer-substrate structure for incident unpolarized or circularly polarized light,

$$
\mathcal{R}_{u}=\frac{1}{2}\left(\mathscr{R}_{p}+\mathscr{R}_{s}\right),
$$

is considered as a function of the system parameters:

$$
\mathcal{R}_{u}=f\left(n_{1}, \zeta_{1}, n_{2}, \zeta_{2} ; n_{3}, k_{3}, \phi\right)
$$

For a given substrate at a given angle of incidence, $n_{3}$, $k_{3}$, and $\phi$ are fixed, and $\mathcal{R}_{u}$ is a function of the refractive indices $n_{1}, n_{2}$ and normalized thicknesses $\zeta_{1}, \zeta_{2}$ of the bilayer. A computer program was developed that 


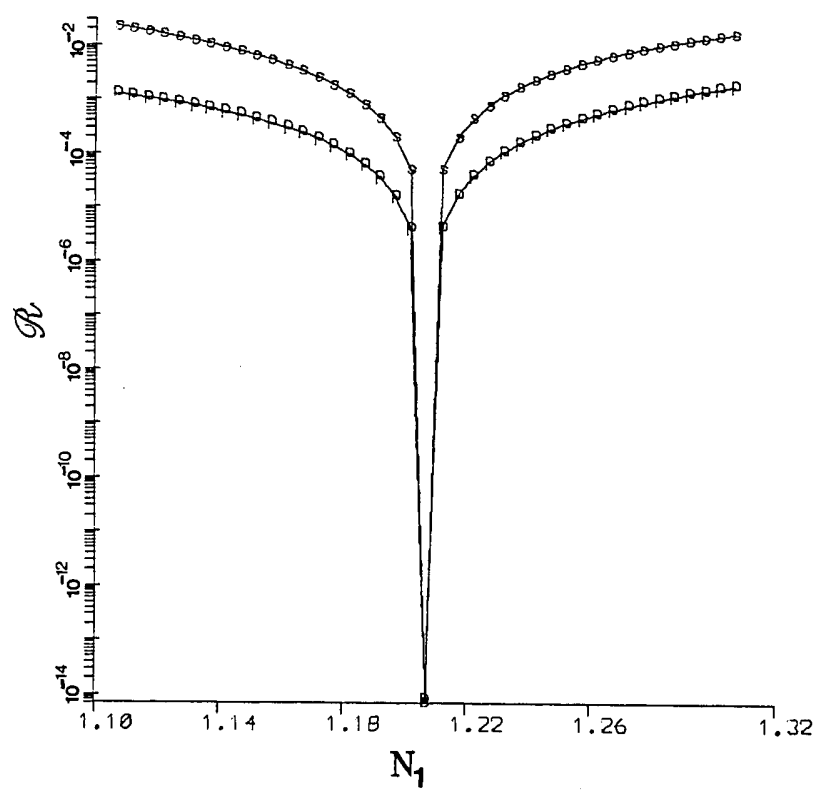

Fig. 3. $\quad p$ and $s$ reflectances (on a logarithmic scale) vs the refractive-index $n_{1}$ of the first film with thickness $d_{1}=2.70920 \mu \mathrm{m}$. The second film has $n_{2}=2.41394$ and $d_{2}=1.14815 \mu \mathrm{m}$. Films 1 and 2 form a bilayer on a Ge substrate $\left(n_{3}=4\right)$ on which $10.6-\mu \mathrm{m}$ radiation is incident from air $\left(N_{0}=1\right)$ at $45^{\circ}$ angle of incidence. When $n_{1}=$ $1.20697, \mathcal{R}_{p}=\mathcal{R}_{s}=0$, and total refraction occurs.

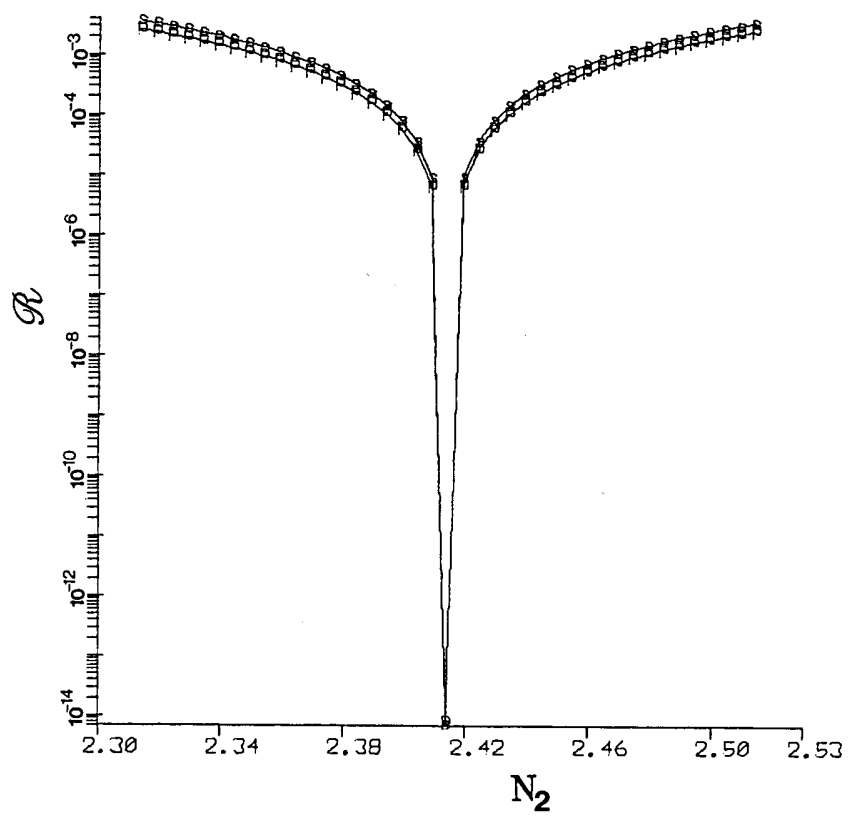

Fig. 4. Same as in Fig. 3 except that now the refractive index of film 2 is being shifted (by \pm 0.1 ) around the design value $n_{2}=2.41394$.

iterates on all four variables $n_{1}, n_{2}, \zeta_{1}, \zeta_{2}$ to minimize $\mathcal{R}_{u}$. Total refraction is considered accomplished when the minimum reflectance $\mathscr{R}_{u \text { min }}$ at the end of iteration is $<10^{-12}$.

As an example, we considered a substrate with complex refractive index $N_{3}=4-j k_{3}$, where the extinction coefficient $k_{3}$ is allowed to increase from 0 to 5 , and an angle of incidence of $45^{\circ}$. Figure 7 shows the (numeri-

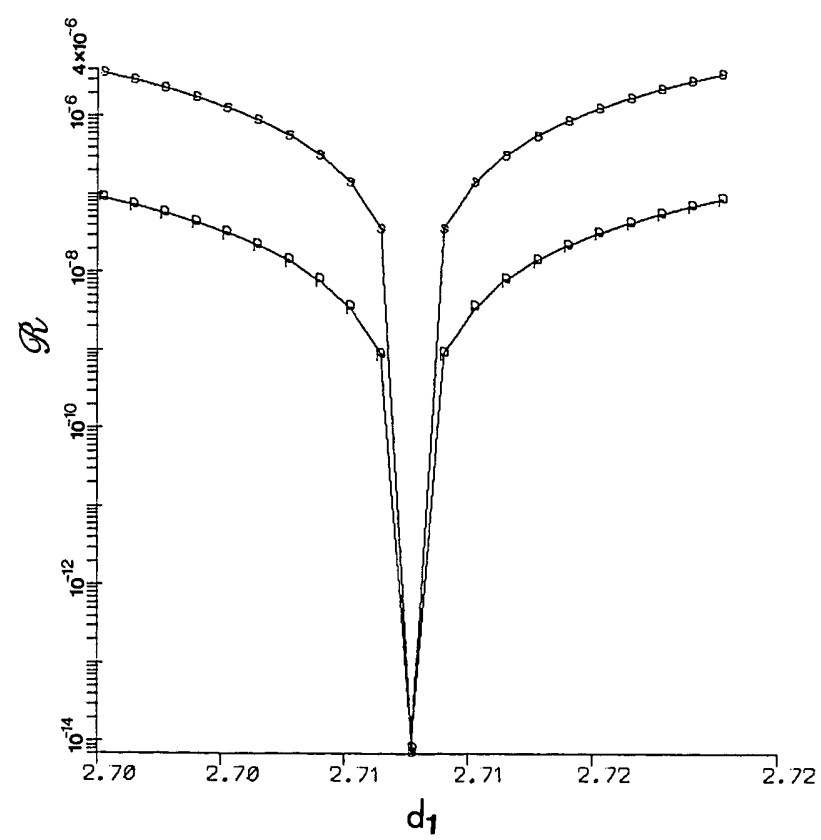

Fig. 5. $\quad p$ and $s$ intensity reflectances (on a logarithmic scale) vs the thickness $d_{1}$ of film 1 of a bilayer $\left(n_{1}=1.20697, n_{2}=2.41394, d_{2}=\right.$ $1.4815 \mu \mathrm{m})$ on a Ge substrate $\left(n_{3}^{\prime}=4\right)$ on which $10.6-\mu \mathrm{m}$ radiation is incident from air $\left(N_{0}=1\right)$ at $45^{\circ}$ angle of incidence. When $d_{1}=$ $2.70920 \mu \mathrm{m}, \mathcal{R}_{p}=\mathscr{R}_{s}=0$, and total refraction takes place.

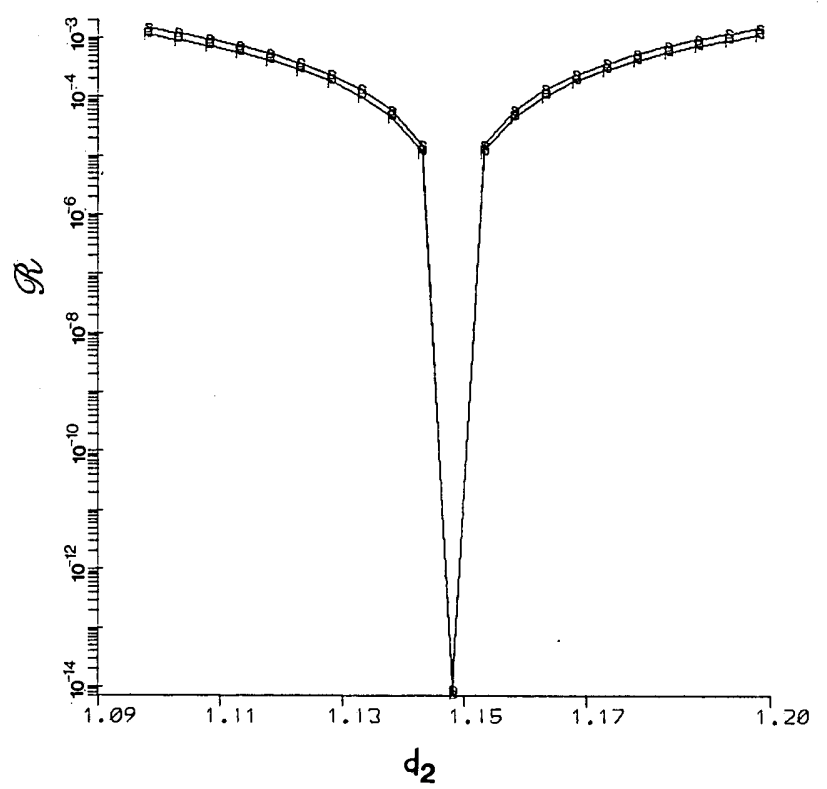

Fig. 6. Same as in Fig. 5 except that the thickness of film 2 is now , being shifted (by $\pm 0.01 \mu \mathrm{m}$ ) around the design value $d_{2}=1.14815 \mu \mathrm{m}$.

cally determined) refractive indices $n_{1}$ and $n_{2}$ of the bilayer plotted against $k_{3}$. As $k_{3}$ increases from 0 to 5 , $n_{1}$ increases slowly and monotonically from 1.20697 to 1.27259 , whereas $n_{2}$ increases appreciably and monotonically from 2.41394 to 4.83923 .

Figure 8 shows the corresponding variation of the normalized thicknesses $\zeta_{1}$ and $\zeta_{2}$ of the two films of the bilayer with $k_{3}$. Both $\zeta_{1}$ and $\zeta_{2}$ decrease monotonically 


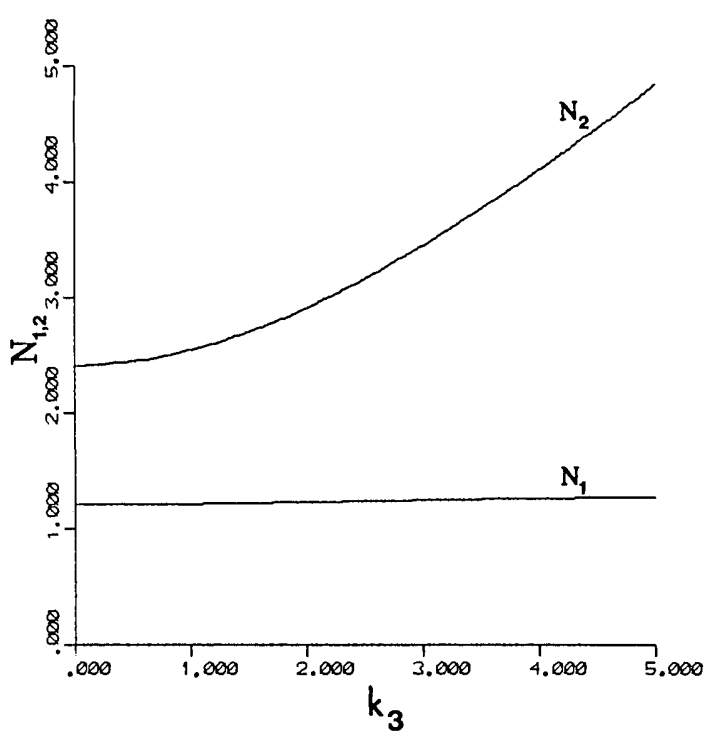

Fig. 7. Refractive indices $n_{1}$ and $n_{2}$ of the two films of a totally refracting bilayer on an absorbing substrate with complex refractive index $N_{3}=4-j k_{3}$ plotted vs the extinction coefficient $k_{3}$. Light is assumed to be incident from air $\left(N_{0}=1\right)$ at $45^{\circ}$ angle of incidence.

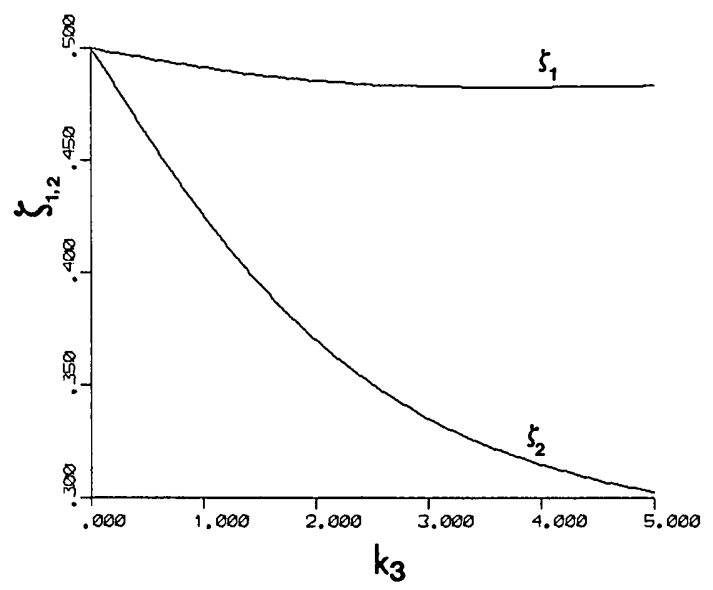

Fig. 8. Normalized thicknesses $\zeta_{1}$ and $\zeta_{2}$ of the two films of a totally refracting bilayer (with refractive indices given by Fig. 7) on an absorbing substrate with complex refractive index $N_{3}=4-j k_{3}$ plotted vs the extinction coefficient $k_{3}$. Light is assumed to be incident from air $\left(N_{0}=1\right)$ at $45^{\circ}$ angle of incidence. as $k_{3}$ increases from the common value of 0.5 at $k_{3}=0$ to 0.483405 and 0.302413 , respectively. Thus the deviation from the quarterwave optical thickness increases as the substrate absorption increases and is much more pronounced for the inner film (film 2) than it is for the outer film (film 1).

Results similar to those shown in Figs. 7 and 8 at other angles of incidence were obtained but are not included here. However, for further illustration, Table I provides numerical data for totally refracting bilayers on a Ti substrate with complex refractive in$\operatorname{dex}^{10} N_{3}=4-j 2$ for $10.6-\mu \mathrm{m} \mathrm{CO}_{2}$-laser radiation at three angles of incidence $\phi=30,45$, and $60^{\circ}$. Table I lists the bilayer refractive indices $\left(n_{1}, n_{2}\right)$ and the normalized $\left(\zeta_{1}, \zeta_{2}\right)$ and actual $\left(d_{1}, d_{2}\right)$ film thicknesses. In all cases the computed minimum reflectance for incident unpolarized light of the bilayer-substrate system is well below $10^{-12}$.

Table II gives the characteristics of totally refracting transparent bilayers on a Si substrate at $45^{\circ}$ angle of incidence for five lines of the $\mathrm{A}_{\mathrm{r}}{ }^{+}$laser and at the 632.8$\mathrm{nm}$ wavelength of the $\mathrm{He}-\mathrm{Ne}$ laser. Listed in that table are the complex refractive index $N_{3}$ of $\mathrm{Si}$, obtained from Aspnes and Studna ${ }^{11}$ by interpolation, the calculated refractive indices $n_{1}$ and $n_{2}$, the normalized thicknesses $\zeta_{1}$ and $\zeta_{2}$, and actual thicknesses $d_{1}$ and $d_{2}$ of the two films of the bilayer. For all cases shown $\mathscr{R}_{u \min }<10^{-12}$ is achieved. The refractive index $n_{1}$ of film 1 is realizable by low-index fluoride coating materials ${ }^{12}$ such as $\mathrm{CaF}_{2}$, whereas $n_{2}$ of film 2 can be obtained by controlling the evaporation conditions of $\mathrm{TiO}_{2}$ as indicated by Pulker et al. ${ }^{13}$

The substrate complex refractive index and angle of incidence determine whether totally refracting bilayers are possible. For certain substrates, we have found that $\mathcal{R}_{u \min }$ cannot be reduced to zero for realistic film refractive indices. For example, for an $\mathrm{Al}$ substrate with $N_{3}=1.212-j 6.924$ at $\lambda=632.8 \mathrm{mn}$, and with the search range of $n_{1}$ and $n_{2}$ limited to $<7$, the lowest value of $\mathcal{R}_{u}$ at $\phi=45^{\circ}$ is $3.13 \%$ and is attained when $n_{1}$ $=7, n_{2}=3.76422, \zeta_{1}=0.49184$, and $\zeta_{2}=0.85006$. This solution, of course, is unacceptable, and three or more layers may be needed for total refraction at oblique incidence.

\section{Summary}

Practical totally refracting transparent bilayers on transparent and absorbing substrates for monochromatic light at oblique incidence have been considered. When the substrate is transparent, the totally refract-

Table I. Characteristics of Totally Refracting Transparent Bllayer Coatings on a TI Substrate $\left(\mathrm{N}_{3}=4-/ 2\right)$ for 10.6- $\mu \mathrm{m} \mathrm{CO}_{2}$-Laser Radiation at Three Angles of Incidence ${ }^{a}$

\begin{tabular}{ccccccc}
\hline$\phi(\mathrm{deg})$ & $n_{1}$ & $n_{2}$ & $\zeta_{1}$ & $\zeta_{2}$ & $d_{1}(\mu \mathrm{m})$ & $d_{2}(\mu \mathrm{m})$ \\
\hline 30 & 1.27008 & 3.02252 & 0.48388 & 0.36073 & 2.19661 & 0.64138 \\
45 & 1.23052 & 2.91216 & 0.48552 & 0.36981 & 2.55520 & 0.69380 \\
60 & 1.17336 & 2.75678 & 0.48792 & 0.38174 & 3.26638 & 0.77304 \\
\hline
\end{tabular}

${ }^{a} \phi$ is the angle of incidence. $n_{1}$ and $n_{2}$ are the refractive indices of films 1 and 2 of the bilayer, whereas $\zeta_{1}, \zeta_{2}$ and $d_{1}, d_{2}$ are the corresponding normalized and actual film thicknesses, respectively. 
Table II. Characteristics of Totally Refracting Transparent Bilayer Coatings on a Si Substrate at $45^{\circ}$ Angle of Incidence for Five $\mathrm{Ar}^{+}$-Laser Lines and the 632.8-nm He-Ne-Laser Line ${ }^{a}$

\begin{tabular}{ccccccc}
\hline $\begin{array}{c}N_{3} \\
(\lambda)\end{array}$ & $n_{1}$ & $n_{2}$ & $\zeta_{1}$ & $\zeta_{2}$ & $d_{1}(\mathrm{~nm})$ & $d_{2}(\mathrm{~nm})$ \\
\hline $\begin{array}{c}5.063-j 3.218 \\
(325 \mathrm{~nm})\end{array}$ & 1.25157 & 3.54333 & 0.48759 & 0.37408 & 76.73 & 17.51 \\
$\begin{array}{c}4.775-j 0.167 \\
(442 \mathrm{~nm})\end{array}$ & 1.22145 & 2.67145 & 0.49894 & 0.49061 & 110.71 & 42.09 \\
$\begin{array}{c}4.639-j 0.141 \\
(454.5 \mathrm{~nm})\end{array}$ & 1.21921 & 2.62776 & 0.49905 & 0.49163 & 114.18 & 44.14 \\
$\begin{array}{c}4.375-j 0.080 \\
(488 \mathrm{~nm})\end{array}$ & 1.21452 & 2.54098 & 0.49939 & 0.49469 & 123.40 & 49.46 \\
$4.168-j 0.053$ & 1.21050 & 2.47163 & 0.49955 & 0.49614 & 134.41 & 55.38 \\
$(528.7 \mathrm{~nm})$ & & & & & 162.20 & 69.59 \\
\hline $\begin{array}{c}3.884-j 0.020 \\
(632.8 \mathrm{~nm})\end{array}$ & 1.20439 & 2.37365 & 0.49980 & 0.49835 & & \\
\hline
\end{tabular}

${ }^{a} N_{3}$ is the complex refractive index of Si at wavelength $\lambda$ obtained by interpolation of the data of Ref. 11. $n_{1}$ and $n_{2}$ are the refractive indices of films 1 and 2 of the bilayer, whereas $\zeta_{1}, \zeta_{2}$ and $d_{1}, d_{2}$ are the corresponding normalized and actual film thicknesses, respectively.

ed wave has the same state of polarization as the incident wave. In this case, the substrate refractive index must be sufficiently high to raise the refractive index of the low-index outer layer to a value that can be realized by a thin solid film. This is illustrated by a Ge substrate with a refractive index of 4 for $10.6-\mu \mathrm{m}$ radiation at $45^{\circ}$ angle of incidence. In this case a $\mathrm{BaF}_{2}-\mathrm{ZnSe}$ bilayer can produce total refraction. The behavior of this bilayer-substrate system as a function of the various design parameters (angle of incidence, wavelength, refractive indices, and thicknesses of the two films) is also studied. The solution to the problem of total refraction by a transparent bilayer is analytically obtained when the substrate is transparent. However, when the substrate is absorbing, the problem is more complicated, and a numerical approach is used. Total refraction is considered attained when the reflectance of the bilayer-substrate structure for incident unpolarized light is reduced to $<10^{-12}$. This was demonstrated for the case of an absorbing substrate with complex refractive index $N_{3}=4-j k_{3}$, where the extinction coefficient $k_{3}$ is allowed to vary from 0 to 5 to represent a range of semiconductors and metals. The normalized thicknesses of the two films of the bilayer differ from 0.5. Hence the films are not of quarterwave of optical thickness, a condition that holds only when the substrate is transparent $\left(k_{3}=0\right)$. Specific examples for which results appear in tables include a Ti substrate $10.6-\mu \mathrm{m}\left(\mathrm{CO}_{2}\right.$-laser $)$ wavelength at three angles of incidence and a Si substrate at several $\left(\mathrm{A}_{\mathrm{r}}{ }^{+}\right.$ and $\mathrm{He}-\mathrm{Ne}$-laser) wavelengths at $45^{\circ}$ angle of incidence.

At the present time, R. M. A. Azzam is at the Département de Physique du Solide, Université de Provence, Marseille, France on a sabbatical leave as a Fulbright Senior Research Scholar and Professeur Associé.

\section{References}

1. See, for example, R. M. A. Azzam, "Single-Layer Antireflection Coatings on Absorbing Substrates for the Parallel and Perpendicular Polarizations at Oblique Incidence," Appl. Opt. 24, 513 (1985).

2. H. Pohlack, "Zum Problem der Reflexionsminderung Optischer Glaser bei Nichtsenkrechtem Lichteinfall," in Jenaer Jahrbuch 1952, P. Gorlich, Ed. (Fischer, Jena, 1952), pp. 103-118.

3. K. Javily and R. M. A. Azzam, "Coating a Transparent or Absorbing Substrate by a Transparent Thin Film for Minimum Reflectance of Unpolarized Light at Oblique Incidence," Optik, in press.

4. P. Baumeister, "The Transmission and Degree of Polarization of Quarter-Wave Stacks at Non-Normal Incidence," Opt. Acta 8, 105 (1961).

5. R. M. A. Azzam and K. Javily, "Antireflecting and Polarizing Transparent Bilayer Coatings on Absorbing Substrates at Oblique Incidence," Appl. Opt. 24, 519 (1985).

6. See, for example, R. M. A. Azzam and N. M. Bashara, Ellipsometry and Polarized Light (North-Holland, Amsterdam, 1977); Eq. (4.186) must be corrected to have the same denominator as Eq. (4.185). This correction follows because $S_{21}$ should be replaced by $S_{11}$ in Eq. (4.170).

7. M. Herzberger and C. D. Salzberg, "Refractive Indices of Infrared Optical Materials and Color Correction of Infrared Lenses," J. Opt. Soc. Am. 52, 420 (1962).

8. P.C. Kemeny, "Refractive Index of Thin Films of Barium Fluoride," Appl. Opt. 21, 2052 (1982).

9. M. E. Pedinoff, M. Braunstein, and O. M. Stafsudd, "Refractive Indices of Materials: 10.6- $\mu \mathrm{m}$ Ellipsometer Measurements," Appl. Opt. 16, 2849 (1977).

10. M. A. Ordal, L. L. Long, R. J. Bell, J. E. Bell, R. R. Bell, R. W. Alexander, Jr., and C. A. Ward, "Optical Properties of the Metals $\mathrm{Al}, \mathrm{Co}, \mathrm{Cu}, \mathrm{Au}, \mathrm{Fe}, \mathrm{Pb}, \mathrm{Ni}, \mathrm{Pd}, \mathrm{Pt}, \mathrm{Ag}$, and $\mathrm{W}$ in the Infrared and Far Infrared," Appl. Opt. 22, 1099 (1983).

11. D. E. Aspnes and A. A. Studna, "Dielectric Functions and Optical Parameters of $\mathrm{Si}, \mathrm{Ge}, \mathrm{GaP}, \mathrm{GaSb}$, InP, InAs, and InSb from 1.5 to $6.0 \mathrm{eV}$," Phys. Rev. B 27, 985 (1983).

12. H. K. Pulker, "Characterization of Optical Thin Films," Appl. Opt. 18, 1969 (1979).

13. H. K. Pulker, G. Paesold, and E. Ritter, "Refractive Indices of $\mathrm{TiO}_{2}$ Films Produced by Reactive Evaporation of Various Titanium-Oxygen Phases," Appl. Opt. 15, 2987 (1976). 